\title{
Viscous hydrodynamic deformation in rapidity distributions of the color glass condensate
}

\author{
Akihiko Monnai* \\ Department of Physics, The University of Tokyo, Tokyo 113-0033, Japan \\ E-mail: monnaiant.phys.s.u-tokyo.ac.jp

\section{Tetsufumi Hirano} \\ Department of Engineering and Applied Science, Sophia University, Tokyo 102-8554, Japan \\ Department of Physics, The University of Tokyo, Tokyo 113-0033, Japan \\ E-mail: hirano@sophia.ac.jp
}

\begin{abstract}
We investigate shear and bulk viscous hydrodynamic deformation on the rapidity distributions of the color glass condensate (CGC) in the relativistic heavy ion collisions at the RHIC and the LHC with a novel numerical and theoretical scheme. The results show that the rapidity distributions of the CGC are clearly deformed due to the entropy flux to forward rapidity and the entropy production from viscosity. The hydrodynamic effects tend to make the distribution steeper at the LHC than at the RHIC. This could be a candidate explanation for the rather surprising excess of multiplicity in the LHC data compared with the bare CGC predictions. The hydrodynamic parameter dependences are also discussed for more quantitative analyses.
\end{abstract}

The Seventh Workshop on Particle Correlations and Femtoscopy

September 20 - 242011

University of Tokyo, Japan

\footnotetext{
${ }^{*}$ Speaker.
} 


\section{Introduction}

The quark-gluon plasma (QGP) [1] created in high-energy heavy ion collisions at the Large Hadron Collider (LHC) is considered to follow the description of a nearly-perfect liquid, as was first discovered at the Relativistic Heavy Ion Collider (RHIC) [2]. The colliding nuclei, on the other hand, are treated within the color glass condensate (CGC) modeling, a description of saturated gluons [3]. It is thus both important and natural to combine these established pictures to obtain a unified understanding of the heavy ion collisions. Relativistic hydrodynamic analyses on the azimuthal anisotropy in particle spectra have revealed that small viscosity is important for explaining the experimental data when the initial conditions are constructed from the CGC picture. The longitudinal evolution of the QGP for the CGC, however, has never been discussed in the context of viscous hydrodynamic models. It has recently been revealed that the bare CGC predictions underestimated the mid-rapidity multiplicity of the $\mathrm{Pb}-\mathrm{Pb}$ collisions at $\sqrt{s_{N N}}=2.76$ $\mathrm{TeV}$ in the LHC experiments [4 5, 6, 7], despite their successes at the RHIC. Estimation of the hydrodynamic effects would shed new light on the efforts to explain the final rapidity distribution in the CGC approach, since the CGC parameter controlling the rapidity dependence would require readjustment. In this study, we develop a viscous hydrodynamic model for the non-boost invariant longitudinal expansion and estimate the modification of the CGC rapidity distributions during the hydrodynamic stage at the RHIC and the LHC settings [8, 9].

\section{Relativistic viscous hydrodynamic model}

The viscous hydrodynamic model is developed with the full second order theory [10,11] which takes account of relaxation effects to preserve causality and stability. In the system with vanishing conserved currents we consider, thermodynamic quantities are given by tensor decomposing the energy-momentum tensor $T^{\mu v}$ in terms of the relativistic flow $u^{\mu}$ as

$$
T^{\mu v}=e_{0} u^{\mu} u^{v}-\left(P_{0}+\Pi\right) \Delta^{\mu v}+W^{\mu} u^{v}+W^{v} u^{\mu}+\pi^{\mu v},
$$

where $\Delta^{\mu v}=g^{\mu v}-u^{\mu} u^{v}$ is the projection operator and $g^{\mu v}=\operatorname{diag}(+,-,-,-)$ the Minkowski metric. $e_{0}$ denotes the energy density, $P_{0}$ the hydrostatic pressure, $W^{\mu}$ the energy dissipation current and $\pi^{\mu v}$ the shear stress tensor. Here we take the flow in the direction of the local energy flux, i.e., the Landau frame is chosen, leading to $W^{\mu}=0$ without the loss of generality. The equations of motion for the hydrodynamic quantities are the energy-momentum conservation $\partial_{\mu} T^{\mu v}=0$ and the constitutive equations for the dissipative currents. Here we employ a full second order viscous hydrodynamics [11] for the constitutive equations by keeping all the second order terms.

We focus on the longitudinal evolution of the hot matter and neglect the transverse dynamics since it is one of the main aims of this study to seek the clue to explaining the discrepancy in multiplicity at the LHC through hydrodynamic analyses. This is in good contrast to the fact that $(2+1)$ dimensional viscous hydrodynamic models are often considered for the analyses of azimuthal anisotropy, neglecting the rapidity dependence of the systems. The constitutive equations in $(1+1)-$ dimensional relativistic coordinates can be written as, using the shear pressure $\pi=\pi^{00}-\pi^{33}$,

$$
D \Pi=\frac{1}{\tau_{\Pi}}\left(-\Pi-\zeta_{\Pi \Pi} \frac{1}{T} \nabla Y_{f}-\zeta_{\Pi \delta e} D \frac{1}{T}+\chi_{\Pi \Pi}^{b} \Pi D \frac{1}{T}+\chi_{\Pi \Pi}^{c} \Pi \nabla Y_{f}+\chi_{\Pi \pi} \pi \nabla Y_{f}\right),
$$




$$
D \pi=\frac{1}{\tau_{\pi}}\left(-\pi+\frac{4}{3} \eta \nabla Y_{f}+\chi_{\pi \pi}^{b} \pi D \frac{1}{T}+\chi_{\pi \pi}^{c} \pi \nabla Y_{f}+\frac{2}{3} \chi_{\pi \pi}^{d} \pi \nabla Y_{f}+\frac{2}{3} \chi_{\pi \Pi} \Pi \nabla Y_{f}\right),
$$

where $Y_{f}$ is the flow rapidity, $T$ the temperature, $\zeta_{\Pi \Pi}$ and $\zeta_{\Pi \delta}$ the bulk viscosities, $\eta$ the shear viscosity, $\tau_{\Pi}$ and $\tau_{\pi}$ the relaxation times, $\chi_{\Pi \Pi}^{b}, \chi_{\Pi \Pi}^{c}, \chi_{\Pi \pi}, \chi_{\pi \pi}^{b}, \chi_{\pi \pi}^{c}, \chi_{\pi \pi}^{d}$ and $\chi_{\pi \Pi}$ the second order transport coefficients. The time- and the space-like derivative operators are defined as $D=$ $\cosh \left(Y_{f}-\eta_{s}\right) \partial_{\tau}+\frac{1}{\tau} \sinh \left(Y_{f}-\eta_{s}\right) \partial_{\eta_{s}}$ and $\nabla=\sinh \left(Y_{f}-\eta_{s}\right) \partial_{\tau}+\frac{1}{\tau} \cosh \left(Y_{f}-\eta_{s}\right) \partial_{\eta_{s}}$ where $\tau$ is the proper time and $\eta_{s}$ the space-time rapidity.

The equation of state $P_{0}=P_{0}\left(e_{0}\right)$ and the transport coefficients are necessary input for hydrodynamic calculations. The equation of state is employed from one of the latest $(2+1)$-flavor lattice quantum chromodynamics results [12]. We introduce models for the transport coefficients because it is incredibly more difficult to estimate them in the first principle methods. The shear viscosity is employed from the conjectured minimum boundary $\eta=s / 4 \pi$ in the Anti-de Sitter space/conformal field theory (AdS/CFT) correspondence picture [13] unless specifically mentioned otherwise. Here $s$ is the entropy density. There are two bulk viscosities $\zeta_{\Pi п}$ and $\zeta_{\Pi \delta e}$ when the linear cross terms are kept, and they can be integrated to the effective bulk viscosity as $\zeta=\left(\zeta_{\Pi \Pi}+c_{s}^{2} \zeta_{\Pi \delta e}\right) / T$ using the energy-momentum conservation and the thermodynamic relations where $c_{s}$ is the sound velocity. The coefficients are estimated based on the non-equilibrium statistical operator method with $\phi^{4}$-theory analyses [14 as $\zeta=(5 / 2)\left[(1 / 3)-c_{s}^{2}\right] \eta$ which is also in accordance with the conjectured minimum boundary condition [15]. The relaxation times and the second order transport coefficients are evaluated in relativistic kinetic theory which yields the relations between the first and the second order coefficients [11]. The results with the hadronic components and those with the quark-gluon components are smoothly connected around the (pseudo-)critical temperature as $\chi=\left[\left(1-\tanh \frac{T-T_{c}}{\Delta T}\right) \chi_{\text {had }}+\left(1+\tanh \frac{T-T_{c}}{\Delta T}\right) \chi_{\mathrm{gg}}\right] / 2$ where $T_{c}=0.17 \mathrm{GeV}$ and $\Delta T=0.02 \mathrm{GeV}$. Note that they are model coefficients to demonstrate the qualitative responses of the systems.

The initial condition for the energy density is constructed from a Monte Carlo model 16, 17] of the Kharzeev-Levin-Nardi approach [18, 19], where the saturation scale at a transverse coordinate $\boldsymbol{x}_{\perp}$ is expressed as

$$
Q_{s, A}^{2}\left(x ; \boldsymbol{x}_{\perp}\right)=2 \mathrm{GeV}^{2} \frac{T_{A}\left(\boldsymbol{x}_{\perp}\right)}{1.53 \mathrm{fm}^{-2}}\left(\frac{0.01}{x}\right)^{\lambda},
$$

where $T_{A}\left(\boldsymbol{x}_{\perp}\right)$ is the thickness function, $x$ the momentum fraction of incident partons and $\lambda$ the parameter which is related with the rapidity dependence of the distribution. The rapidity distribution tends to become steeper with increasing $\lambda$. Typically $\lambda \sim 0.2-0.3$ is motivated by the deep inelastic scattering data from the Hadron Electron Ring Accelerator, and we employ $\lambda=0.28$ which is considered to be compatible with the data from the RHIC [17]. The initial distribution $e\left(\tau_{0}, \eta_{s}\right)$ is employed from the transverse energy distribution averaged over the overlapping area $\left(1 / S_{\text {area }}\right) d E_{T} / d y$ for the most central 0-5\% events by matching the momentum rapidity $y$ with the space-time rapidity $\eta_{s}$ and by taking into account the contribution of the low $p_{T}$ gluons $\left(0.1<p_{T}<3 \mathrm{GeV}\right)$ which should form the locally thermalized medium. The initial time is set as $\tau_{0}=1 \mathrm{fm} / c$. The initial conditions for the dissipative currents, on the other hand, are not well-known. In this study they are chosen as $\Pi\left(\tau_{0}, \eta_{s}\right)=\pi\left(\tau_{0}, \eta_{s}\right)=0$ because assuming the same energy-momentum tensors is necessary for qualitative comparison between ideal and viscous hydrodynamic results. This would 

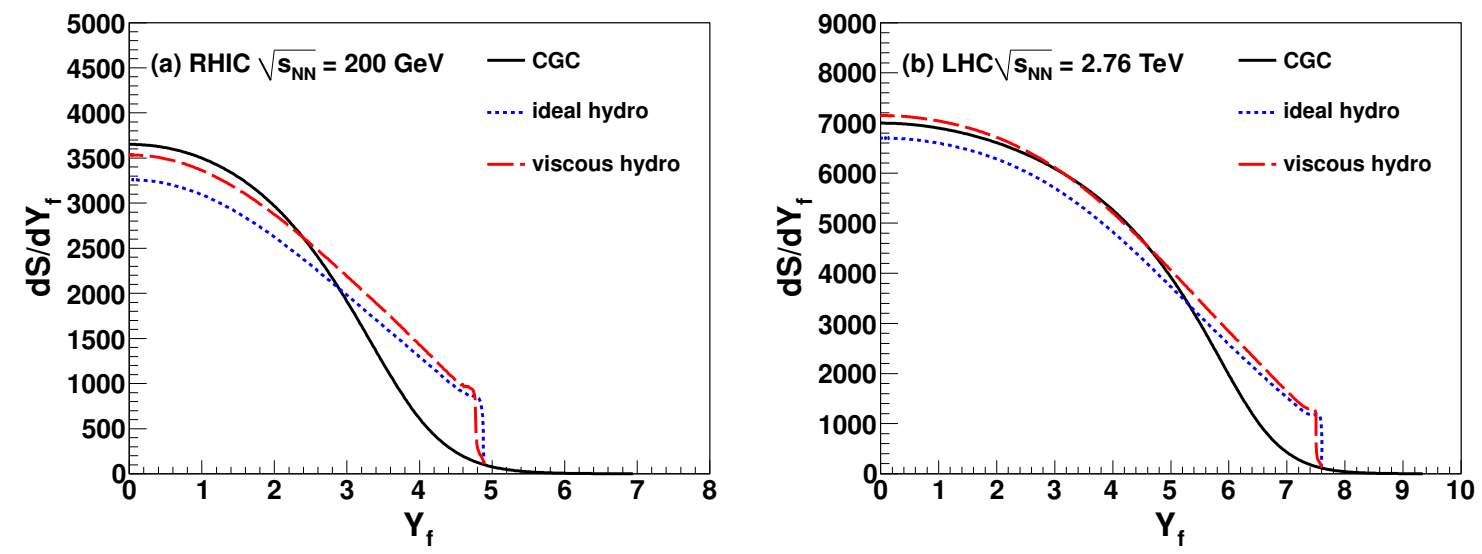

Figure 1: The distributions for (a) the $\sqrt{s_{N N}}=200 \mathrm{GeV}$ Au-Au collisions at the RHIC and (b) the $\sqrt{s_{N N}}=$ $2.76 \mathrm{TeV} \mathrm{Pb}-\mathrm{Pb}$ collisions at the LHC. The solid lines are the initial CGC distributions, the dotted lines the ideal hydrodynamic ones and the dashed lines the viscous hydrodynamic ones.

also reduce possible overestimation of viscous effects coming from the unknown factors. The initial flow is the Bjorken flow $Y_{f}\left(\tau_{0}, \eta_{s}\right)=\eta_{s}$. Note that this boost-invariance in the flow is broken as soon as the hydrodynamic evolution is started.

The rapidity distribution is estimated through the entropy distribution per the flow rapidity $d S / d Y_{f}$ [20]. The two quantities are related through $d N_{\mathrm{ch}}^{\text {hydro }} / d y \approx(2 / 3) \times(1 / 3.6) \times d S / d Y_{f}$, because the dimensional analysis suggests that the ratio of entropy density to number density is temperature independent in the relativistic massless gas limit and the flow rapidity in a fluid element on average corresponds to the momentum rapidity. The flow rapidity distribution, therefore, is the closest pure hydrodynamic quantity to the rapidity distribution which does not involve other model assumptions. We do not consider hadronization because the initial distribution is given as an energy/entropy distribution which of course is not of hadrons, and one has to choose a corresponding quantity in order to compare the final hydrodynamic result with the initial CGC distribution. Here we develop an entropic freeze-out scheme and construct the rapidity distribution from the entropy current coming out of the isothermal hyper-surface at $T_{f}=0.16 \mathrm{GeV}$.

\section{Results}

The rapidity distributions for the Au-Au collisions at $\sqrt{s_{N N}}=200 \mathrm{GeV}$ in the RHIC and the $\mathrm{Pb}-\mathrm{Pb}$ collisions at $\sqrt{s_{N N}}=2.76 \mathrm{TeV}$ in the LHC are shown in Fig. 1. One sees that the CGC distributions are visibly modified in the hydrodynamic stage for both cases. The ideal hydrodynamic results exhibit flattening effects on the distributions caused by the entropy density carried to forward rapidity with flow due to the non-boost invariant nature of the systems. The viscous hydrodynamic results, on the other hand, show enhancement of the rapidity distributions compared with the ideal hydrodynamic ones which can be attributed to the entropy production from viscosity. The competition of the two effects - the outward entropy flux and the entropy production - determines the overall magnitude of hydrodynamic effects. The cutoff-like structures before the beam rapidities are due to the fact that the thermal smearing effect at the hadronic stage is not included, which 

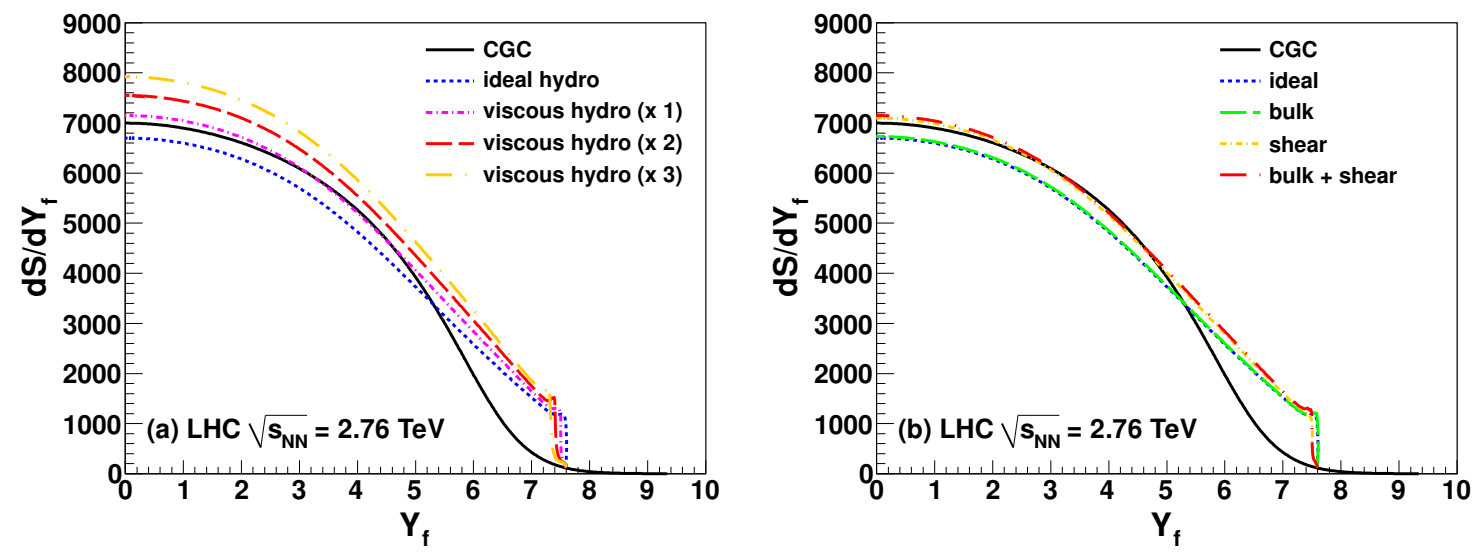

Figure 2: The parameter dependences of the rapidity distributions for $\sqrt{s_{N N}}=2.76 \mathrm{TeV} \mathrm{Pb}-\mathrm{Pb}$ collisions at the LHC. The solid line corresponds to the initial CGC distribution. (a) The dotted, short dash-dotted, dashed and long dash-dotted lines are the ideal hydrodynamic result and the viscous hydrodynamic results with $\eta / s=1 / 4 \pi, 2 / 4 \pi$ and $3 / 4 \pi$, respectively. (b) The dotted, dashed, short dash-dotted and long dashdotted lines correspond to ideal, bulk, shear and bulk-and-shear viscous hydrodynamic results, respectively.

of course does not affect the discussion of the hydrodynamic modifications here. Quantitatively speaking, the slope of the distribution around mid-rapidity after viscous hydrodynamic modification becomes slightly steeper at the LHC while it becomes flatter at the RHIC. This would be one of the possible factors for explaining the underestimation of the bare CGC approach at the LHC, because if, as the current parameter sets suggest, the distribution is flattened at the RHIC, then the true initial distribution has to be steeper, i.e., the parameter $\lambda$ in the CGC model should be larger. Since the hydrodynamic modification at the LHC is almost cancelled or shows slight enhancement, the larger $\lambda$ translates into the enhancement of the multiplicity at mid-rapidity.

The hydrodynamic parameter dependences of the distribution with the LHC settings are also explored. In Fig. 2 (a), the viscous hydrodynamic results with $\eta / s=1 / 4 \pi, 2 / 4 \pi$ and $3 / 4 \pi$ are shown. Note that bulk viscosity $\zeta$ is also increased along with $\eta$. One can see that the entropy production almost linearly increases with the viscous coefficients, which is consistent with that fact that viscous corrections are perturbation to the local equilibrium. In the cases where the viscous effect is larger and the hydrodynamic effects are almost cancelled at the RHIC, $\lambda$ would be unaffected but again the slope of the LHC distribution becomes enhanced due to the extra entropy production. Finally in Fig. 2 (b), the rapidity distributions with ideal, bulk viscous, shear viscous and bulk-and-shear viscous hydrodynamic effects are separately plotted. For the current parameter settings, the effect of shear viscosity is dominant over that of bulk viscosity for the hydrodynamic evolution, while the latter is not entirely invisible. Note that the results do not suggest that bulk viscosity can be neglected a priori, especially since the $\zeta / \eta$ ratio is dependent on a model.

\section{Conclusions}

We developed a viscous hydrodynamic model with the full second order theory for the nonboost invariant longitudinal evolution. The numerical estimations show that the rapidity distribu- 
tions of the CGC are modified due to (i) the flattening effect induced by the entropy flux to forward rapidity and (ii) the enhancing effect caused by the entropy production from viscosity. The distribution is more flattened at the RHIC than at the LHC in the hydrodynamic evolution, which is a candidate explanation for the CGC under-prediction of the mid-rapidity multiplicity because when the CGC parameters are readjusted so that the final hydrodynamic result explains the experimental data at the RHIC, one has enhancement in the particle production at the LHC. This suggests that viscous hydrodynamic estimation with non-boost invariant flow is indispensable for the qualitative discussion of multiplicity in the CGC approach. The explorations on hydrodynamic parameter dependences support the above conclusions. It would be important to investigate the CGC parameter dependences through the distributions as functions of rapidity and centrality, to introduce final state interactions and to estimate the effects of transverse dynamics for more quantitative discussion.

\section{Acknowledgments}

The authors acknowledge fruitful discussion with Yasushi Nara. The work of A.M. was supported by JSPS Research Fellowships for Young Scientists. The work of T.H. was partly supported by Grant-in-Aid for Scientific Research No. 22740151.

\section{References}

[1] K. Yagi, T. Hatsuda and Y. Miake, Camb. Monogr. Part. Phys. Nucl. Phys. Cosmol. 23 (2005) 1.

[2] T. Hirano, N. van der Kolk and A. Bilandzic, Lect. Notes Phys. 785 (2010) 139.

[3] F. Gelis, E. Iancu, J. Jalilian-Marian and R. Venugopalan, Ann. Rev. Nucl. Part. Sci. 60 (2010) 463.

[4] K. Aamodt et al. [ALICE Collaboration], Phys. Rev. Lett. 105 (2010) 252301.

[5] K. Aamodt et al. [ALICE Collaboration], Phys. Rev. Lett. 106 (2011) 032301.

[6] S. Chatrchyan et al. [CMS Collaboration], JHEP 1108 (2011) 141.

[7] G. Aad et al. [ATLAS Collaboration], arXiv:1108.6027 [hep-ex].

[8] A. Monnai and T. Hirano, Phys. Lett. B 703 (2011) 583.

[9] A. Monnai and T. Hirano, J. Phys. G: Nucl. Part. Phys. 38 (2011) 124168.

[10] W. Israel and J. M. Stewart, Annals Phys. 118 (1979) 341.

[11] A. Monnai and T. Hirano, Nucl. Phys. A 847 (2010) 283.

[12] S. Borsanyi et al., JHEP 1011 (2010) 077.

[13] P. Kovtun, D. T. Son and A. O. Starinets, Phys. Rev. Lett. 94 (2005) 111601.

[14] A. Hosoya, M. a. Sakagami and M. Takao, Annals Phys. 154 (1984) 229.

[15] A. Buchel, Phys. Lett. B 663 (2008) 286.

[16] H. J. Drescher and Y. Nara, Phys. Rev. C 75 (2007) 034905.

[17] H. J. Drescher and Y. Nara, Phys. Rev. C 76 (2007) 041903.

[18] D. Kharzeev and E. Levin, Phys. Lett. B 523 (2001) 79.

[19] D. Kharzeev, E. Levin and M. Nardi, Nucl. Phys. A 730 (2004) 448 [Erratum-ibid. A 743 (2004) 329].

[20] K. Morita, S. Muroya, C. Nonaka and T. Hirano, Phys. Rev. C 66 (2002) 054904. 